\title{
Health care leadership development and training: progress and pitfalls
}

\author{
This article was published in the following Dove Press journal: \\ Journal of Healthcare Leadership \\ 12 February 2016 \\ Number of times this article has been viewed
}

\author{
Roberta E Sonnino ${ }^{1,2}$ \\ 'Department of Surgery, Division \\ of Pediatric Surgery, Wayne State \\ University School of Medicine, \\ Detroit, MI, USA; ${ }^{2}$ RES Coaching LLC, \\ Locust Hill, VA, USA
}

\begin{abstract}
Formal training in the multifaceted components of leadership is now accepted as highly desirable for health care leaders. Despite natural leadership instincts, some core leadership competencies ("differentiating competencies") must be formally taught or refined. Leadership development may begin at an early career stage. Despite the recognized need, the number of comprehensive leadership development opportunities is still limited. Leadership training programs in health care were started primarily as internal institutional curricula, with a limited scope, for the development of faculty or practitioners. More comprehensive national leadership programs were developed in response to the needs of specific cohorts of individuals, such as programs for women, which are designed to increase the ranks of senior women leaders in the health sciences. As some programs reach their 20th year of existence, outcomes research has shown that health care leadership training is most effective when it takes place over time, is comprehensive and interdisciplinary, and incorporates individual/institutional projects allowing participants immediate practical application of their newly acquired skills. The training should envelop all the traditional health care domains of clinical practice, education, and research, so the leader may understand all the activities taking place under his/her leadership. Early career leadership training helps to develop a pipeline of leaders for the future, setting the foundation for further development of those who may chose to pursue significant leadership opportunities later in their career. A combination of early and mid-to-late career development may represent the optimal training for effective leaders. More training programs are needed to make comprehensive leadership development widely accessible to a greater number of potential health care leaders. This paper addresses the skills that health care leaders should develop, the optimal leadership development concepts that must be acquired to succeed as a health care leader today, some resources for where such training may be obtained, and what gaps are still present in today's system.
\end{abstract}

Keywords: leadership competencies, leadership traits, leadership skills, health care, training

\section{Introduction}

Health care organizations are complex environments that require strong, comprehensive, and collaborative leadership. Over the past 15-20 years, awareness of the importance of leadership in health care and of formal leadership training has increased dramatically. Historically, advancement to leadership positions in medicine was based on the candidate's academic or clinical accomplishments, with no expectation of knowledge in the so-called differentiating competencies, such as finances, team building, communication skills, and emotional intelligence. The concept of leadership has evolved from the top-down, paternalistic model, where the leader is in complete control and demands performance from others, to a more collaborative approach,
Correspondence: Roberta E Sonnino RES Coaching LLC, 185 Brandon Point Road, Locust Hill, VA 23092, USA

Tel +l 8042869174

Email RESCoachingLLC@gmail.com 
where the leader helps his/her team develop a vision and empowers them to accomplish the stated goals. ${ }^{1}$ Many have suggested that formal training in the multifaceted components of leadership is necessary and should begin at an early career stage, ${ }^{2-4}$ yet still today, the number of comprehensive leadership training opportunities, at any career level, is limited.

While the literature on the topic of leadership has increased substantially, reports on comprehensive health care leadership training programs (including interdisciplinary programs) are still scarce, no doubt a reflection of the paucity of such programs. ${ }^{2}$ Most publications on the subject of leadership training concentrate on a specific sector of health care, or a stage of professional training, and describe group-centered curricula that are often devised for internal constituencies. Several reviews of the literature on leadership training programs have appeared, ${ }^{5,6}$ but few describe the major national, comprehensive health care leadership training opportunities. ${ }^{7}$

Leadership training programs in health care were started primarily as internal institutional curricula, with a limited scope, for the development of faculty or practitioners. ${ }^{8}$ More comprehensive leadership programs were developed in response to the needs of specific cohorts of individuals. Programs for women (both comprehensive and specialtyspecific) $)^{9-11}$ were among the first to appear in an attempt to increase the ranks of senior women leaders in the health sciences. ${ }^{12}$ The long-existing programs such as the Executive Leadership in Academic Medicine (ELAM) program have shown that ${ }^{10,13,14}$ the health care leadership training is most effective when it takes place over time, is comprehensive and interdisciplinary, and incorporates individual/institutional projects with immediate practical application of newly acquired skills. ${ }^{2,15}$

Particularly important is the notion that the training should envelop all the traditional health care domains of clinical practice, education, and research, so the leader may understand all the activities taking place under his/her leadership and whether or not he/she is personally engaged in all of them. A leader must be able to engage the various interprofessional communities within the hospital, academic affiliate, or health care system.

Leadership training for students and medical residents is a rising topic of discussion, ${ }^{2}$ with some programs implementing short leadership retreats for rising senior or chief residents ${ }^{4,16}$ and others incorporating leadership training in their regular trainee curriculum. If uniformly implemented, these early career programs would be of great value in developing a pipeline of leaders for the future. Early career programs would likely be insufficient to create the leadership skills necessary for senior career opportunities but may establish the foundation for continued development. More development programs are needed to make comprehensive leadership training more widely accessible. Better coordination among disciplines, and expanded availability of comprehensive leadership development opportunities, would result not only in better prepared senior health care leaders but also in improved interactions between hospital administrators, practicing professionals, and academicians, with a culture of true collaborative or shared leadership.

This paper will discuss the skills that health care leaders should develop, the optimal leadership training concepts to succeed as a health care leader today, some resources for where such training may be obtained, and what gaps are still present in today's system.

\section{Types of leadership}

The historical definition of leadership in the dictionary ${ }^{17}$ was "the position or function of a leader, a person who guides or directs a group", with synonyms ${ }^{18}$ that included "administration", "management", and "control". This definition has evolved over time, and today, we recognize different styles of leadership, each with their own definitions. In health care, three of these types are prevalent and most identified: ${ }^{19}$

- Transactional leaders, who work within the boundaries and the existing standards of the organization. They are usually not risk takers, but focus on efficiency, control, stability, and predictability.

- Transformational leaders, who raise one another to higher levels of motivation, making changes and shaping the future.

- Servant leaders, who focus on the service aspect first as they have a natural tendency to help others. ${ }^{20}$

Each of these types has its place in health care, but transformational and servant leaders are more likely to help the institution advance, while transactional leaders are most qualified to maintain the status quo.

Today's leaders require two general types of behaviors: "task" behaviors and "relationship" behaviors. Task behaviors allow the individual to accomplish his/her goals and enable leaders to guide others in achieving their objectives. Relationship behaviors involve the ability to interact with peers and subordinates in a way that all feel comfortable with themselves, with each other, and their specific setting. ${ }^{19}$ A leader may be more task oriented in certain situations and more relationship oriented in others. Therefore, individuals are, by necessity, becoming more aware of their own leadership styles and the way they communicate, usually through feedback from others. Physicians, for example, are not 
usually trained to concentrate on leadership or think about their own behavioral style. ${ }^{21}$ By the nature of their profession, they tend to focus on outcomes rather than the processes involved in achieving those outcomes. Yet, leadership is an intrinsic part of the practice of medicine, even in the interactions with patients and their families. Gabel ${ }^{22}$ examined how all physicians take on leadership roles at some time in their careers, whether formally or informally. He discussed the characteristics of formal and informal leaders and concluded that it is important to expand the scope of leadership training so that both types of leaders are included.

Different health care leaders may arrive at their positions via different personal paths. Rogers ${ }^{19}$ published an analysis of the communication and leadership styles of health care leaders in each major area within an academic health center, that is, medicine, nursing, and administration. She studied the importance of linking leadership styles to individual professions. She concluded that physicians, nurses, and administrators must have an increased awareness of self and individual leadership style and that each of these health care leaders must be engaged in practices of reflection.

In nursing, reaching a leadership role is often an expectation or at least an aspiration for a large number of individuals. It should therefore be no surprise that the nursing profession has embraced leadership training earlier than others in health care. ${ }^{23,24}$ Likewise, hospital administrators are usually "big picture" leaders, having come up through the administrative ranks. They likely have managerial experience, so the transition to leadership is fairly natural, as long as they are able to also have vision in addition to their managerial skills. One cannot, however, assume that these individuals possess all the traits that will make them effective leaders in a large and complex health care system: the development of integrated leadership processes throughout health care delivery systems is needed. ${ }^{23}$

For physicians, the transition to becoming true modern leaders is a major accomplishment, ${ }^{25}$ often requiring a move outside their comfort zone. In fact, many cringe at the thought of having to be a leader. ${ }^{23}$ Senior physicians, in particular, do not always have a system's perspective, which is an important competency for a health care leader. Arroliga et al state that failure to train our healthcare leaders could have a long-term negative impact on society. He argues that the traditional means of selecting leaders (by virtue of age, productivity or other academic skills) was inadequate, as these individuals simply emulated their predecessors, but had no formal development of the personal and professional qualities and skills required by a leader. ${ }^{2}$
Thankfully, this may be changing as educational standards for trainees evolve. In 2002, the Accreditation Council on Graduate Medical Education (ACGME) launched their outcomes project, a competency initiative that included six core competencies that residency programs use to evaluate their residents. One of these is systems-based practice. These competencies, now included in the ACGME accreditation system as performance milestones, ${ }^{26}$ began the task of introducing this topic into medical education, albeit at the graduate level. As a result, many medical schools have elected to incorporate these skills in their medical student curriculum as well. Hopefully, the trend of investing in the leadership growth of physicians from their earliest development will allow them to acquire the skills to become better collaborative leaders, with a vision for the entire realm of health care.

\section{Traits of health care leaders}

Contrary to the old image, today's leader must possess the skills of listening, empathy, awareness, persuasion, conceptualization, foresight, stewardship, commitment to the growth of people, and building community. Stoller ${ }^{27}$ observed that health care leaders must also possess commitment, integrity, altruism, and authenticity. He listed some of the skills and traits that physician leaders, in particular, must have or acquire. These include a technical knowledge of insurance and reimbursement issues, how to balance expense with quality of patient care, health care regulations (including the Affordable Care Act), legal issues in health care and public policy, problem-solving skills, communication skills, emotional intelligence, and a commitment to lifelong learning. In his editorial in the American Journal of Medicine, Alpert ${ }^{28}$ defines qualities that are important for leaders. These include commonly mentioned themes such as equity, justice, role modeling, work ethic, balancing work and personal life, organization, and prioritization. Developing all these skills may be a challenge for many individuals.

In the early years of recognition that health care professionals required some form of leadership training, degree programs such as MPH and MBA were thought to be the solution. ${ }^{24}$ These programs provide knowledge in specific areas (global health, epidemiology, financial skills, etc), together with managerial and administrative skills. But experience has shown that these additional degrees do not necessarily turn an individual into a leader. Managers, administrators, and leaders are not one and the same. While an individual may encompass all three of these descriptors, often that is not the case. As described by Curtis et al, ${ }^{29}$ managers administer, maintain, control, have a short-term view, and initiate. Other 
managerial roles include planning and budgeting, organizing resources, and problem solving. Leaders, on the other hand, "innovate, develop, inspire, challenge the status quo, and focus on a long-term vision". Kotterman ${ }^{30}$ defines management as dealing with procedures, practices, and complexity and leadership as dealing with change.

While the skills acquired through various Master's programs are indeed highly desirable in many leadership roles, comprehensive leadership programs are necessary for the development of broad leadership skills - the "differentiating competencies." A program at Duke ${ }^{31}$ for medical residents who have already obtained graduate management training (eg, MD-MBA) combines the benefits of formal MBA training with training and experiential learning in the leadership competencies and may represent a new model for early career leadership development.

Souba $^{32}$ discussed the changes that have occurred in health care leadership over the years. He described the shift in traits from those that made an individual a leader in the "old" days to those accepted today. Business and administrative acumen, a foreign concept for a clinical leader of the past, is now a necessity. The ability to engage others in creating a common vision and building teamwork is in stark contrast with the old image of the leader as a despot, commanding from above. Strong communication skills were always appreciated but are often lacking in the leader: lack of communication today is likely to derail a leader. Possibly, the most dramatic change in required skills is the concept of emotional competence - the ability to look within oneself, recognizing not only the feelings of others but also one's own. The concepts of awareness and authenticity have gained wide acceptance. Empathy and the ability to develop others through mentoring and coaching are, today, among the traits that truly differentiate a leader from the rest.

\section{The need for formal, comprehensive health care leadership development}

While some individuals are "born leaders" with good instincts, some formal leadership training in the differentiating competencies is needed - many skills must be learned or refined. This may include rules, laws, governance, or the personal competencies that are not innate in all. Leaders who believe that they can do it without any formal training often succeed for some time but eventually will encounter critical situations that they are not prepared to handle alone. Then they urgently seek the resources to help them succeed, often too late to salvage a career. The fact is that when the need for a leader in a given role presents itself, taking an individual who has demonstrated mastery of his/her profession's skills and expecting her/him to become a leader intuitively, is no longer a satisfactory (or successful) model. We need a pipeline of emerging leaders, both in the purely clinical health care realm and in academia, who have already mastered the additional skills through formal experiential training and will have greater odds of success when asked to step into a new leadership role. It would be preferable for all if training in the key knowledge and skills were more easily accessible and therefore acquired before a major institutional and career failure dictate the need for more education.

These needs are global: the Foundation for Advancement of International Medical Education and Research (FAIMER), ${ }^{33}$ based on the ELAM model ${ }^{10}$ and cofounded by the Founding Director of ELAM, demonstrated that leadership and management can be taught across cultures and that this has enhanced the ability of the leaders in the served countries to maintain alignment with their local needs, developing trust in their own abilities and avoiding persistent dependency on other countries. This is a unique example that appears to validate the principles of leadership training developed for American health care as they apply to the global health care community. ${ }^{10,34}$

Warren and Carnall ${ }^{35}$ from the UK noted that their national health care system has also neglected leadership training for physicians. They encouraged physicians to develop skills and a level of understanding that go beyond technical expertise in their specialty, including the ability to create and communicate their vision and set clear direction. Warren and Carnall concluded that leadership training programs were most successful when the participants were given the opportunity, during the program, to work on some of the "real time" challenges they faced at their home institution. They also concurred with the opinion that programs spanning longer periods of time, allow the participants to absorb and reflect on their new knowledge, and incorporate it in their daily activities.

\section{What should training include?}

Because leadership development is needed for all the professions from which health care leaders emerge (medical, dental, public health, nurses, allied health providers, and administrators), the ideal curriculum would be interdisciplinary and applicable to as many as possible of these groups. Such a program should include essential and universal leadership skills (conflict management, negotiation, financial skills, etc), as well as strategies to develop personal traits. Table 1 lists a compilation of the essential elements of leadership development and the competencies considered the most important skills to be developed in health care leaders. 
Some general concepts are universally important for leadership development. For an effective program that will give leaders insight into themselves and others, pretesting for learning style and personality characteristics are a must.

Table I Optimal elements and competencies of health care leadership development

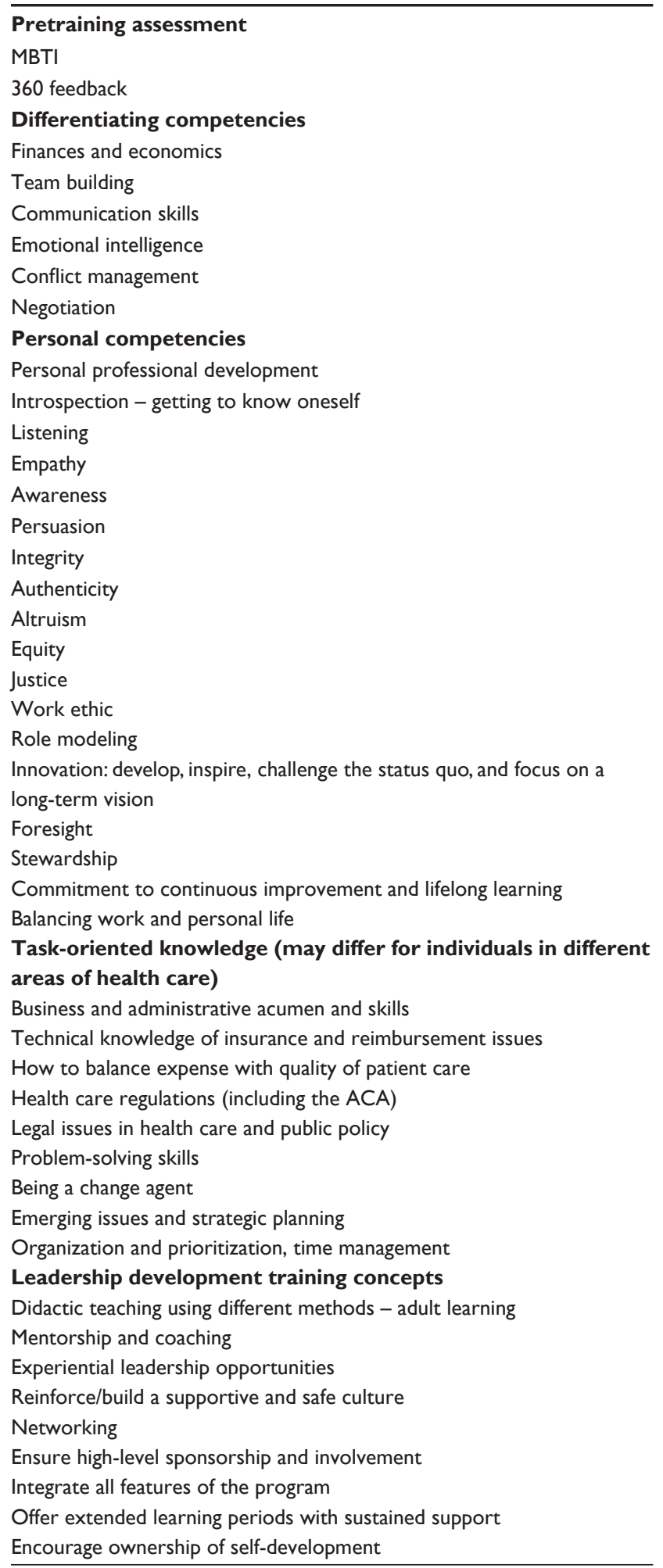

Abbreviations: MBTI, Myers Briggs type indicator; ACA, Affordable Care Act.
These include identifying each individual's Myers Briggs type indicators and completion of formal 360 feedback evaluations. Learning how to use the information obtained from these assessments is a key to develop the insight to one's own characteristics, as well as learning to interact effectively with individuals very similar or very different from oneself.

An ideal leadership development program would include a basic curriculum of general, comprehensive health care concepts, presented with diverse methodologies, including didactic teaching, mentorship and coaching, and experiential leadership opportunities. In addition, there should be specific elements for each individual's area of leadership, be it hospital administration, a clinical setting, or academia. Even more detailed specialty-specific topics may be appropriate, if feasible. For example, surgeons, operating room nurses, and allied health providers need to learn operating room-related leadership skills and information, whereas clinic nurses and outpatient-based physicians and pharmacists need to learn skills specific to their ambulatory setting. Basic researchers have an entirely different set of skills required to run a major research program, and educators need the proper tools to lead curriculum development, innovative practices in education, etc.

Training about leadership styles and situational leadership should be a component of the curriculum for emerging health care leaders, allowing them to understand and be able to interact with individuals with different styles from their own.

Among the competencies that should be included in most comprehensive leadership curricula, the most significant include finances and economics, emerging issues and strategic planning, personal professional development, adaptive leadership, conflict management, time management, ethical considerations, and personal life balance. In addition, developing a well-defined project that will have an impact on the institution provides a practical, on-the-job application of skills learned that are therefore more likely to become ingrained.

Blumenthal et $\mathrm{al}^{3}$ suggested that the common elements of effective leadership development programs include reinforcing or building a supportive culture, ensuring highlevel involvement and mentorship, using a variety of learning methods, offering extended learning periods with sustained support, encouraging ownership of self-development, and committing to continuous improvement. These concepts are echoed by others, such as The University of Minnesota Medical School Emerging Physician Leaders Program (EPLP). ${ }^{34}$ This 3-year program for young physicians applies principles that are of universal value: 
- Leaders develop personal resilience through continual learning. Lifelong learning is essential to success as a professional.

- Adult learning includes discovering the personal meaning of ideas. Opportunities for applying learning to one's experience must be meaningful, varied, and frequent. Learning shared with a cohort of fellow learners enhances the discovery process: adults can learn as much from each other as they do from formal instruction. Learning is also enhanced when participants share common work experiences.

- While learning is unique to each person, learners need consistent methods of reviewing and improving learning outcomes.

- Learning is reinforced by mentoring and coaching and becomes embedded when participants are able to utilize what they have learned in a timely manner.

Mentoring ${ }^{36}$ deserves special attention. It is rightfully considered a key component of leadership training, especially at the emerging leader level. The guidance of a more experienced individual allows the emerging leader to safely experiment with his/her developing style and knowledge. A mentor is a "a trusted counselor or guide" 17 who will direct the mentee in navigating institutional politics and processes, help with specific tasks such as manuscript or grant preparation, and keep the individual on a steady path of upward career development. Mentors are not reserved for emerging leaders: one is never too old or too senior to benefit from good mentorship.

The next step, which in my opinion should be a required component of senior leader development, is exposure to executive coaching. Coaching differs from mentoring in that it is directed at enhancing performance in specific areas. It is goal oriented and may be a relatively short-term process, although many successful leaders avail themselves of a coach for their entire careers. A key distinction is that the coach is there not to direct (as a mentor may do) but to provide a "third opinion", by asking pertinent questions and helping the leader arrive at the recognition of the right steps or decisions to make. ${ }^{37}$ Velsor et al state that individuals who receive coaching remain responsible for communicating their learning needs to their coach, and for implementing the plans developed together. It is their responsibility to assure that the topics of discussion are appropriate to their needs and address any feedback they may have received. The coach does not set the agenda, but is there to assure that it is developed properly. ${ }^{38}$

This is the ultimate example of "sustained support" that is purely within the control of the leader.
Finally, the environment in which the training occurs plays an important role. It must be understood to be "safe", a place where everything is open for discussion, without repercussions, honesty and candor, is welcome and respected and, above all, will remain confidential within the confines of the training partners. A long-term benefit of longer training programs, not to be underestimated, is the networking and peer mentoring that inevitably develops. It is often stated that "it is lonely at the top": the relationships developed during these types of intense training often result in a continuation of that safe environment long after the program has been concluded. What emerge are life-long relationships and networks of trusted peers that can provide strong, safe, and valued support.

\section{Examples of leadership development programs}

Many programs have been developed to enhance culture- or situation-specific skills. Training may occur in different formats, ranging from self-directed to team training or formal curricula of variable duration. Of the programs described in the literature, a large number are directed toward graduate medical education (residencies), mostly in response to requirements by the accrediting body (ACGME). Most of these programs have short and intense components (eg, day-long retreats) that may be followed by small elements disseminated throughout the training period. ${ }^{4,16,31}$

Devising a curriculum at the professional school level is a greater challenge: the curriculum is tightly planned; therefore, such early career programs are most successful when integrated in the core curriculum, over the course of the entire duration of the degree program or planned during breaks from school. Most of the integrated programs are targeted to students who are enrolled in dual degree programs, such as $\mathrm{MD} / \mathrm{PhD}^{39}$ and $\mathrm{MD} / \mathrm{MBA}$ or $\mathrm{MD} / \mathrm{MPH},{ }^{40}$ and therefore already have the expectation of a longer overall timeline. Other programs for health care students in medicine, dentistry, and nursing have been described..$^{23,41-43}$ Public health schools have also started to address this need by offering specific leadership tracks beyond the core curriculum for public health that by its nature already includes some leadership components. ${ }^{44}$

Institution-based leadership programs directed toward faculty (usually at the junior level) are emerging with increasing frequency. Some examples include the University of California at San Diego National Center of Leadership in Academic Medicine, ${ }^{45}$ the Emerging Physician Leaders Program $^{34}$ at the University of Minnesota Medical School, the University of Virginia Leadership in Academic Medicine 
Program, ${ }^{46}$ the multidisciplinary Woodruff Leadership Academy at Emory, ${ }^{47}$ the Primary Care Faculty Leadership Fellowship for Family Medicine at the University of Texas Health Science Center at San Antonio, ${ }^{48}$ The University of Washington Teaching Scholars Program, ${ }^{49}$ and the Cleveland Clinic Academy. ${ }^{26}$ The latter program has a structure that includes freestanding "a la carte" courses on a variety of leadership topics available to all physicians, nurses, and administrators, as well as leadership development workshops for all incoming chief residents and developmental coaching for newly appointed chairs, thereby providing training at different career levels.

In more recent years, a number of specialty organizations have also developed specialty-specific leadership programs. A few examples include the American College of Surgeons Leadership Course for Surgeons, ${ }^{50}$ covering topics such as the attributes of a leader, aligning values and leading change, building and maintaining team effectiveness, and leading oneself. The American Academy of Pediatrics, together with the Johnson and Johnson Pediatric Institute, developed the Pediatric Leadership Alliance's Young Pediatric Leaders for the 21st Century Training Program to provide leadership skills to pediatricians who are $<40$ years old or have $<5$ years in practice. ${ }^{51}$ The Association of American Medical Colleges offers a variety of development programs annually, including those for Early and Mid Career Women, Minorities, Aspiring Leaders, GME Development, and Executive Seminars for Associate Deans and Department Chairs. ${ }^{11}$

These types of targeted programs are important and play key roles in the development of students, trainees, junior faculty, and practitioners. Nevertheless, there is a need for more national-level interdisciplinary and comprehensive leadership training programs: these are still relatively scarce as are leadership training opportunities for senior career individuals who have already attained administrative and leadership roles. National programs have access to a broader cohort of participants from diverse backgrounds and attract some of the best individuals in the country. This enhances the experience as participants and faculty bring their diverse experiences to the program and the networking element is expanded to a broad area. Programs available at this time include a selection of excellent general leadership training programs (albeit mostly not directed toward health care) offered by the Center for Creative Leadership, based in Greensboro, NC; $;^{37,38,52,53}$ the Harvard Macy Program for Educators in Health Professions; ${ }^{54}$ and the Harvard leadership programs ${ }^{55}$ (Program for Chiefs of Clinical Services, Leadership Development for Physicians in Academic Health Centers, The International Leadership
Development Program for Physicians, Leadership Strategies for Evolving Health Care Executives). These programs are intense and relatively short (1-2 weeks duration).

Few comprehensive leadership programs have undergone formal evaluation. Stoller ${ }^{26}$ noted that "true return on investment analysis of a leadership development program has yet to be done." One exception is the ELAM. Now in its 20th year, ELAM is the most comprehensive national (and international) program available today, albeit specific to the academic sector, and open only to women. ELAM enrolled its first class in 1995 and has to date trained almost 900 senior-level women in academic medicine, dentistry, and public health. Evaluation has been an integral component of the program since its onset..$^{10,12-14}$ The most recent report ${ }^{10}$ shows that

Leadership skills and knowledge increase after participation; a greater proportion of ELAM alumnae advance to higher levels of academic leadership than do comparison groups; and medical and dental school deans view the ELAM program as having a positive impact both on their schools and on participants. ${ }^{10}$

In fact, $63.5 \%$ of the ELAM graduates report having achieved positions of department chair or greater. These included, at the time of publication, 14 of the 26 women deans at US accredited medical schools, seven of the eleven women deans at US dental schools, and one of the 13 women deans at US public health schools. Twenty-eight ELAM alumnae have held or currently hold positions of vice president, provost, or president of an academic institution of higher learning, and seven hold equivalent leadership positions in organizations outside academia (foundations, pharmaceutical industry). The ELAM model has also been applied to two independent leadership programs: FAIMER,$^{33}$ as mentioned earlier, and Nonprofit Executive Leadership Institute, ${ }^{56}$ through the Bryn Mawr College Graduate School of Social Work and Social Research. The documented outcomes from ELAM suggest that similar programs, open to a wider population of senior health care leaders, would help in resolving the deficiencies described by many reports in the literature.

\section{Benefits and pitfalls of leadership training}

Over the past 20 years, we have made considerable progress in the field of leadership development.

Leadership is a common topic of conversation in health care today, and there is an increasing body of literature and awareness of leadership development needs and opportunities. 
As noted earlier, a very significant step forward is that outcomes of comprehensive leadership training programs are being evaluated both in the academic environment ${ }^{10}$ and when the emphasis is on clinical providers. ${ }^{49}$

Graduates of these development programs are highly recruited nationally, and their knowledge is spread to diverse geographic areas. As a consequence of the experience during a development program, there is greater acceptance of executive and leadership coaching. This has finally created a culture where leaders no longer feel that they have to "go it alone." Hopefully, this will also help to erase the longstanding perception that coaching is primarily a remedial tool, when one is "in trouble." The availability of input from a trusted coach may prevent egregious errors, potentially saving a career, such as the situation of a department chair who wished to discuss reorganization of the clinical service with the hospital CEO. She had not discussed it with her boss, the medical school dean. Her coach reminded her that blindsiding a superior is never a good idea. The chair therefore shared her ideas with the dean before discussing them with the CEO and discovered that the dean had different ideas. The chair changed her strategy to align with the dean's vision, which resulted in a plan that was acceptable to all. Given the impact of the reorganization, had she moved forward without first consulting the dean, it is likely that she would have been removed from her chair position.

Formal leadership development resources are growing in number and quality: the benefits of formal training are many and usually evident, albeit not always documented with objective data. Institutions and health care in general directly benefit by the increasing numbers of individuals already in leadership roles with formal training. With the rise of programs for students and postgraduate residents and fellows, a pipeline of physicians, nurses, and administrators with some formal leadership training is being created: this bodes well for succession planning and sustained organizational success of our health care systems. There are immediate benefits as well: development programs directed toward junior-level health care providers and/or faculty members have the added benefit of enhancing participants' career and organizational satisfaction that often results in better retention, even if not all choose to advance to leadership roles..$^{45,57}$ Leadership development courses can also be innovation incubators for the organizations: several programs, such as ELAM, the Emerging Physician Leaders Program, and the Cleveland Clinic Academy require that participants develop and implement a project to enhance institutional performance.
Significant individual benefits include personal growth, career satisfaction and advancement, and, very importantly, networking: participants who spend significant periods of time learning together often develop a special camaraderie, which encourages ongoing collaboration and synergy among colleagues and institutions.

Relying only on leadership training programs to develop new leaders is not free of risk to both the individual and the institution. First, not all leadership programs address the differentiating leadership competencies (especially emotional intelligence) that set true transformational and servant leaders apart, giving them the personal tools needed to move health care forward. Programs may not include key components that are covered by the more comprehensive curricula. Reliance on such a program alone may not truly prepare the individual for a proposed leadership role: in these cases, it may be wise for an individual to fill any gaps by participating in more than one training opportunity.

A second pitfall is the cost of training in times of limited resources: even short programs require significant resources and time away from work for both course faculty and participants. This becomes even more significant for a curriculum that follows the recommendation of providing opportunities to practice and implement new knowledge during the program itself. Leadership theory (from didactic teaching) alone is not sufficient to "make" a leader nor is a practice/apprenticeship without the proper knowledge background: both must take place concurrently for concepts to become imprinted, but this requires that the program last an entire year or more (such as ELAM and EPLP). Obviously, this impacts costs significantly, whether covered by the institution or the individual. In either case, a substantial sum is at risk, should the participant not develop into a true leader, or not secure a position where the new skills may be applied. To my knowledge, there has not yet been an analysis comparing the costs of offering a program (including everything from faculty and administrative support to food, facilities, and time away from work) with the revenue savings that may result from the enhanced skills of the participants.

Stoller ${ }^{21,26}$ asked the questions: "What is the evidence that acquiring these competencies ties to better organizational and/ or personal performance? What are the best strategies and learning formats in which to cultivate these competencies in emerging physician-leaders, and, at what point in one's training are the competencies best developed?" Research carried out on the institutions and participants in the ELAM program gives some insight into the first question, ${ }^{10}$ with data that support the conclusion that both the organization 
and the individuals do in fact perform better. The authors found that "for the post-ELAM survey, composites of leadership knowledge and skills showed significant differences from presurvey means for all composites except Diversity Competence" (where fellows had initially rated their competence very high). The authors concluded that the data suggest that "confidence in knowledge and readiness for leadership increased between program start and the posttest, and that this increase is a predictable outcome of participation in the program." Their quantitative and qualitative research also found that both the women who participated in the ELAM program and the institutions served by ELAM graduates had received significant benefit from the program. From their data, the concept of a "Leadership continuum" emerged, encompassing four elements such as 1) preparing for leadership, 2) transitioning into leadership, 3) sustaining success in a leadership position, and finally 4) transitioning again into another leadership position.

Stoller's second question is more difficult to answer. Strategies and learning formats are as diverse as the programs themselves. However, some commonalities seem to emerge: programs that adopt diverse learning formats, adaptable to the different personality types of the participants and those that are spread out over time are more likely to allow the principles to be "lived" and experienced in such a way that they become second nature. Furthermore, as noted earlier, programs that require the implementation of a project of importance to both the trainee and his/her institution allow for a safe first experience in using the skills that will be important in future leadership roles.

The optimal timing of training is less clearly defined: Should leadership training take place early during professional school? ${ }^{28}$ If so, should it continue in some form through postgraduate and early faculty/practitioner experience? ${ }^{46} \mathrm{Or}$, should leadership training be reserved for later phases of a career, when experiences have already created a subconscious leadership mentality, clinical skills are mature and the health care providers can devote their full attention to developing new leadership competencies? ${ }^{10}$ We have not yet answered these important questions, but it is possible, even likely, that a combination of both may be ideal. Early career training happens at a time when many experiences that will shape the individual have not yet occurred. This limits the individual's ability to completely comprehend and absorb those skills. However, generic competencies are readily understood and embedded at that time. Training as a more seasoned professional works upon a personal infrastructure where many of the building blocks are already in place. It is logical to assume that taken in sequence, both would have the greatest impact, over the course of career maturation, in developing highly skilled health care leaders. Such a model does not exist yet, but it is possible that some of the students who were exposed to early career training programs will eventually participate in senior leadership development courses as well. Tracking those individuals, if feasible, may provide valuable information.

\section{Conclusion}

We have made a great deal of progress in the acceptance and implementation of leadership development programs, although there is no uniformity of career stage, timing, duration, or curriculum. With some broad, comprehensive programs reaching maturity and evaluation data covering a 20 -year period, there is more information available and more formally trained individuals in our health care systems, who are able to disseminate and role model the information they have learned. There are data to support the optimal methodology, and opportunities are increasing, although not yet reaching all individuals who might benefit. With resources and expertise, these obstacles may be overcome in reasonable time. Health care systems, academic institutions, and the practitioners themselves would be well served to find ways to make formal leadership development accessible and part of the routine career evolution for emerging health care leaders.

\section{Disclosure}

The author reports no conflict of interest in this work.

\section{References}

1. Serio CD, Epperley T. Physician leadership - a new model for a new generation. Fam Pract Manag. 2006;13(2):51-54.

2. Arroliga AC, Huber C, Myers JD, Dieckert JP, Wesson D. Leadership in health care for the 21 st century: challenges and opportunities. Am J Med. 2014;127(3):246-249.

3. Blumenthal DM, Bernard K, Bohnen J, Bohmer R. Addressing the leadership gap in medicine: residents' need for systematic leadership development training. Acad Med. 2012;87(4):513-522.

4. Blumenthal DM, Bernard K, Fraser TN, Bohnen J, Zeidman J, Stone VE. Implementing a pilot leadership course for internal medicine residents: design considerations, participant impressions, and lessons learned. $B M C$ Med Educ. 2014;14:257-267.

5. Rosenman ED, Shandro JR, Ilgen JS, Harper AL, Fernandez R. Leadership training in health care action teams: a systematic review. Acad Med. 2014;89(9):1295-1306.

6. Careau E, Biba G, Brander R, et al. Health leadership education programs, best practices, and impact on learners' knowledge, skills, attitudes, and behaviors and system change: a literature review. J Healthc Leadersh 2014;4:39-50.

7. Sonnino RE. Professional development and leadership training opportunities for healthcare professionals. Am J Surg. 2013;206:727-731.

8. Morahan PS, Kasperbauer D, McDade SA, et al. Training future leaders of academic medicine: internal programs at three academic health centers. Acad Med. 1998;73(11):1159-1168. 
9. Graham EA, Wallace CA, Stapleton FB. Developing women leaders in medicine at the grass roots level: evolution from skills training to institutional change. $J$ Pediatr. 2007;151:1-2.

10. Morahan PS, Gleason KA, Richman RC, Dannels S, McDade SA. Advancing women faculty to senior leadership in u.s. academic health centers: fifteen years of history in the making. $J$ Women High Educ. 2010;3(1):137-162.

11. AAMC. Leadership programs [homepage on the Internet]; 2015. Available from: https://www.aamc.org/meetings/; http://www.cvent.com/ events/2015-early-career-women-faculty-professional-developmentseminar/event-summary-d959a1 ec7b4340429f75555ae213bf93.aspx; https:/www.aamc.org/members/leadership/catalog/323118/mid-career womenfacultyprofessionaldevelopmentseminar.html; http://www.cvent. com/events/2015-executive-development-seminar-for-aspiring-leaders/ event-summary-86571dcd2a6d4cb687f3eb333e61ebce.aspx; http:// www.cvent.com/events/2015-gme-leadership-development-course-partiii-sponsored-by-the-aamc-group-on-resident-affairs/event-summary56cac6d5b2d14167b9cbe30c30e7a912.aspx. Accessed April 28, 2015.

12. Richman RC, Morahan PS, Cohen DW, McDade SA. Advancing women and closing the leadership gap: the executive leadership in academic medicine (ELAM) program experience. JWomens Health Gend Based Med. 2001;10(3):271-277.

13. McDade SA, Richman RC, Jackson GB, Morahan PS. Effects of participation in the executive leadership in academic medicine (ELAM) program on women faculty's perceived leadership capabilities. Acad Med. 2004;79(4):302-309.

14. Dannels SA, Yamagata H, McDade SA, et al. Evaluating a leadership program: a comparative, longitudinal study to assess the impact of the executive leadership in academic medicine (ELAM) program for women. Acad Med. 2008;83(5):488-495.

15. Savage GT, Duncan WJ, Knowles KL, Nelson K, Rogers DA, Kennedy KN. Interprofessional academic health center leadership development: the case of the University of Alabama at Birmingham's Healthcare Leadership Academy. Appl Nurs Res. 2014;27:104-108.

16. Lee MT, Tse AM, Naguwa GS. Building leadership skills in paediatric residents. Med Educ. 2004;38:545-576.

17. Merriam-Webster Dictionary (Encyclopaedia Britannica Co); 2015. Available from: http://www.merriam-webster.com/dictionary/ leadership. Accessed August 21, 2015.

18. Thesaurus.com. 2015. Available from: http://www.thesaurus.com/ browse/leadership?s=t. Accessed August 21, 2015.

19. Rogers R. Leadership communication styles: a descriptive analysis of health care professionals. J Healthc Leadersh. 2012;4:47-57.

20. Greenleaf RK. Servant Leadership - A Journey into the Nature of Legitimate Power and Greatness. Paulist Press Mahwah, NJ; 1977.

21. Stoller JK. Developing physician-leaders: a call to action. J Gen Intern Med. 2009;24(7):876-878.

22. Gabel S. Expanding the scope of leadership training in medicine. Acad Med. 2014;89:848-852.

23. Scott ES. Perspectives on healthcare leader and leadership development. $J$ Healthc Leadersh. 2010;2:83-90.

24. Schwartz RW, Pogge CR, Gillis SA, Holsinger JW. Programs for the development of physician leaders: a curricular process in its infancy. Acad Med. 2000;75(2):133-140.

25. Souba WW. The being of leadership. Philos Ethics Humanit Med. 2011;6:5.

26. ACGME. Milestones [homepage on the Internet]; 2015. Available from: http://www.acgme.org/acgmeweb/tabid/430/ProgramandInstitutionalAccreditation/NextAccreditationSystem/Milestones.aspx. Accessed April 18, 2015.

27. Stoller JK. Recommendations and remaining questions for health care leadership training programs. Acad Med. 2013;88(1):12-15.

28. Alpert JS. Leadership in Academic Medicine. Am J Med. 2010; 123(12):1071-1072.

29. Curtis AE, Vries JD, Sheerin FK. Developing leadership in nursing: exploring core factors. Br J Nurs. 2011;20(5):306-309.
30. Kotterman J. Leadership versus management: what's the difference? J Qual Particip. 2006;29(2):13-17.

31. Ackerly DC, Sangvai DG, Udayakumar K, et al. Training the next generation of physician executives: an innovative residency pathway in management and leadership. Acad Med. 2011;86(5):575-579.

32. Souba WW. The new leader: new demands in a changing, turbulent environment. J Am Coll Surg. 2003;197(1):79-87.

33. Burdick WP. Global faculty development: lessons learned from the foundation for advancement of international medical education and research (FAIMER) initiatives. Acad Med. 2014;89(8): 1097-1099.

34. Rothenberger D. Emerging Physician Leaders Program [homepage on the Internet]; 2015. Available from: http://www.surg.umn.edu/eplp/ about/index.htm. Accessed April 21, 2015.

35. Warren OJ, Carnall R. Medical leadership: why it's important, what is required, and how we develop it. Postgrad Med J. 2011;87:27-32.

36. Hawkins JW, Fontenot HB. Mentorship: the heart and soul of health care leadership. J Healthc Leadersh. 2010;2:31-34.

37. Riddle D: Leadership Coaching - When It's Right and When You're Ready. Center for Creative Leadership Ideas Into Action Guidebooks. Greensboro, NC: CCL; 2008.

38. Velsor EV, McCauley CD, Ruderman MN. The Center for Creative Leadership Handbook of Leadership Development. 3rd ed. San Francisco, CA: Jossey-Bass; 2010.

39. Ciampa EJ, Hunt AA, Arneson KO, et al. A workshop on leadership for MD/PhD students. Med Educ Online. 2011;16:7075-7083.

40. Crites GE, Ebert JR, Schuster RJ. Beyond the dual degree: development of a five-year program in leadership for medical undergraduates. Acad Med. 2008;83(1):52-58.

41. Taichman RS, Parkinson JW. Where is leadership training being taught in US dental schools? J Dent Educ. 2012;76(6):713-720.

42. Taichman RS, Parkinson JW, Nelson BA, NordquistB, Ferguson-Young DC, Thompson JF Jr. Program design considerations for leadership training for dental and dental hygiene students. J Dent Educ. 2012;76(2):192-199.

43. Coleman MB, Blatt B, Greenberg L. Preparing students to be academicians: a national student-led summer program in teaching, leadership, scholarship, and academic medical career-building. Acad Med. 2012;87(12):1734-1741.

44. Uno H, Zakariasen K. Public health leadership education in North America. J Healthc Leadersh. 2010;2:11-15.

45. Wingard DL, Garman KA, Reznik V. Facilitating faculty success: outcomes and cost benefit of the UCSD National Center of Leadership in Academic Medicine. Acad Med. 2004;79(10):S9-S11.

46. Sanfey H, Harris I, Pollart S, Schwartz A. Evaluation of the University of Virginia Leadership in Academic Medicine Program. Teach Learn Med. 2011;23(4):347-358.

47. Korschun HW, Redding D, Tea GL, Johns MME. Realizing the vision of leadership development in an academic health center: the Woodruff Leadership Academy. Acad Med. 2007;82(3):264-271.

48. Tysinger JW, Katerndahl DA, Freeman J. The primary care faculty leadership fellowship for family medicine. Acad Med. 2001; 76(5):573-574

49. Robins L, Ambrozy D, Pinsky LE. Promoting academic excellence through leadership development at the University of Washington: the teaching scholars program. Acad Med. 2006;81(11):979-983.

50. Pellegrini C. Surgeons as Leaders: From Operating Room to Boardroom [homepage on the Internet]. Chicago, IL: American College of Surgeons; 2015. Available from: https://www.facs.org/ education/division-of-education/courses/surgeonsasleaders. Accessed February 5, 2015.

51. Leslie LK, Miotto MB, Liu GC, et al. Training young pediatricians as leaders for the 21st century. Pediatrics. 2005;115(3):765-773.

52. Center for Creative Leadership. Addressing the leadership gap in the healthcare sector: what's needed when it comes to leader talent? CCL's White Paper Series. Greensboro, NC; 2011. 
53. Browning HW, Torain DJ, Patterson TE. Collaborative Healthcare Leadership. A six-part model for adapting and thriving during a time of transformative change. CCL's White Paper Series. Greensboro, NC; 2011.

54. Harvard-Macy. Program for Educators in Health Professions [homepage on the Internet]; 2015. Available from: http://www.harvardmacy. org/programs/Programs-Educators.aspx. Accessed April 30, 2015.

55. Harvard Leadership Programs. Available from: http://www.hmscme.net $/ 352117 /$ edu/programs.cfm?CSID $=$ PCCS0116. Accessed November 30, 2015.
56. NELI. Nonprofit Executive Leadership Institute [homepage on the Internet]; 2003. Available from: http://www.brynmawr.edu/neli/. Accessed April 21, 2015.

57. Garman KA, Wingard DL, Reznik V. Development of junior faculty's self-efficacy: outcomes of a National Center of Leadership in Academic Medicine. Acad Med. 2001;76(10):S74-S76.

Journal of Healthcare Leadership

\section{Publish your work in this journal}

The Journal of Healthcare Leadership is an international, peer-reviewed, open access journal focusing on leadership for the health profession. The journal is committed to the rapid publication of research focusing on but not limited to: Healthcare policy and law; Theoretical and practical aspects healthcare delivery; Interactions between healthcare and society and evidence-based practices;
Dovepress

Interdisciplinary decision-making; Philosophical and ethical issues; Hazard management; Research and opinion for health leadership; Leadership assessment. The manuscript management system is completely online and includes a very quick and fair peer-review system. Visit http://www.dovepress.com/ testimonials.php to read real quotes from published authors.

Submit your manuscript here: http://www.dovepress.com/journal-of-healthcare-leadership-journal 